\title{
Sistema de controle de velocidade sincronizada entre dois veículos agrícolas
}

\author{
Synchronized speed control system between two agricultural vehicles
}

\author{
Rodrigo Fernando Galzerano BaldoI Paulo Sergio Graziano Magalhães ${ }^{\text {II }}$
}

\section{RESUMO}

Este trabalho apresenta uma proposta para o controle automático de velocidade entre dois veículos que necessitam trafegar em paralelo durante operações agrícolas. É descrito o desenvolvimento e os testes de campo de um sistema de controle de velocidade para um trator escravo baseado na velocidade de um trator mestre, utilizando um controlador desenvolvido em lógica fuzzy. Para esses testes, os tratores foram instrumentados com GPS, encoder, computador e transmissor de radiofrequência, sendo instalado ainda no trator escravo um motor de passo para o controle da velocidade de deslocamento. Para avaliar o sistema, realizaram-se dois testes: no primeiro, a resposta do trator escravo à variação de velocidade foi avaliada a partir de simulações de variação na velocidade de entrada; no segundo, foram utilizados dois tratores variando a velocidade do trator mestre e observando a resposta do trator escravo. No primeiro teste, o trator escravo acompanhou a simulação da variação da velocidade com um erro quadrático médio (EQM) não significativo e erro médio percentual (EMP) máximo de 1,3\%. No segundo teste, o trator escravo acompanhou a variação de velocidade do trator mestre com o (EMP) de deslocamento variando em módulo de 0,2\% a $2,9 \%$.

Palavras-chave: piloto-automático, trator, controle fuzzy, colheita mecanizada.

\section{ABSTRACT}

This paper presents a proposal for automatic speed control of vehicles that requires working in synchronism with each other during agricultural operations. It describes the development and field tests of a control system for a slave tractor based on the master tractor speed, using a fuzzy controller. For the tests the master tractor was instrumented with GPS, encoders, computer and radio transmitter. The slave tractor was instrumented in the same way, and included also a stepper motor to act on the tractor throttle. To evaluate the system two tests were conducted. In the first, the master tractor speed was simulated using only the slave tractor. In the second test, two tractors were used varying the master tractor speed and registering the slave tractor response. In the first test the slave tractor followed the speed variation simulated with a mean square error (MSE) non-significant and a maximum mean percentage error (MPE) of $1.3 \%$. In the second test the slave tractor followed the master tractor speed variation with a displacement (MPE) ranging in magnitude from $0.2 \%$ to $2.9 \%$.

Key words: auto-pilot, tractor, fuzzy control, mechanized harvester.

\section{INTRODUÇÃO}

Uma das causas de perda de matéria-prima durante a colheita é a falta de sincronismo entre a colhedora e o trator que traciona a carreta de recolhimento de biomassa, especialmente nas colhedoras que não têm capacidade de armazenamento, como as colhedoras de forragem, café e cana-de-açúcar. Além das perdas de matéria-prima, existe também uma diminuição da capacidade operacional efetiva quando o transbordo se adianta em relação à colhedora, obrigando o operador do trator a realizar manobras para realinhar os equipamentos na posição ideal de trabalho.

Nas duas últimas décadas, o uso de sistemas de controle eletrônico em máquinas agrícolas

'Faculdade de Ciências Aplicadas (FCA), Universidade Estadual de Campinas (UNICAMP), R. Pedro Zaccaria, 1300, 13484-350, Limeira, SP, Brasil. E-mail: rodrigo.baldo@fca.unicamp.br. Autor para Correspondência.

"Faculdade de Engenharia Agrícola (FEAGRI), UNICAMP, Campinas, SP, Brasil. 
vem aumentando, graças à ampla difusão dos sistemas de satélite de posicionamento global (GPS) e ao avanço da informática e da eletrônica. Observa-se um crescente interesse na adoção de pilotos automáticos e isso se dá pelo fato de que este sistema diminui o estresse do operador e possibilita ainda uma maior confiabilidade no trabalho noturno (BERGLUND \& BUICK, 2005). A fadiga mental sobre estes operadores não é causada apenas pela monotonia do trabalho, mas também devido ao estresse criado pela necessidade de dirigir com precisão dentro de linhas estreitas sem causar dano à vegetação, mantendo um ritmo de trabalho adequado.

À medida que a tecnologia avança, informações mais ricas e precisas estão sendo incorporadas aos sistemas inteligentes de veículos agrícolas, exigindo que as informações de controle sejam rapidamente tratadas para garantir uma resposta confiável e rápida do sistema. Operações agrícolas automatizadas são reguladas por sistemas de controle com retroalimentação e cuja execução de loops incluem sensores para as variáveis sob controle (ROVIRAMÁS, 2010).

Atualmente, os pilotos automáticos comerciais não controlam a velocidade dos veículos e sim a direção, baseando-se em referências préestabelecidas. Os sistemas de controle de velocidade e direção para veículos agrícolas autônomos são geralmente baseados em teorias de controle que empregam modelos proporcional integral derivativo (PID), por ser mais simples e fácil de implementar. Esse tipo de controle requer que o modelo matemático do sistema seja conhecido, o que nem sempre está disponível. Para aplicações mais complexas, com parâmetros e que apresentam incertezas e comportamentos não lineares, o emprego da lógica fuzzy para controle tem apresentado melhores resultados que os controladores PID (KODAGODA et al., 2002).

Além dos pilotos-automáticos, observa-se que há interesse em desenvolver soluções específicas mais complexas, como, por exemplo, detectar a posição e orientação do veículo em relação à cultura em tempo real, como apresentado por KISE et al. (2005). Os autores utilizaram duas câmeras como sensores de visão para guiar o trator entre as fileiras de soja. O sistema de acionamento automático consistia de uma válvula solenoide direcional proporcional de controle e um sensor de ângulo potenciométrico na roda para a execução de circuito fechado de controle de direção. A análise quantitativa dos resultados indicaram que a raiz do erro quadrático médio (EQM) do desvio lateral entre a trajetória realizada e a trajetória de referência foi de $0,03 \mathrm{~m}$, a uma velocidade de $2,5 \mathrm{~m} \mathrm{~s}^{-1}$, mas os autores afirmaram que poderiam melhorar a acurácia do sistema através da adoção de métodos de controle de direção mais robusto, como feed-forward, mais PID e controle fuzzy. SUBRAMANIAN et al. (2006) também desenvolveram um sistema de controle automático PID de direção para tratores trafegando em pomares de citros, utilizando sensor de visão, radar laser e encoders. Segundo os autores, o EQM de posicionamento foi, em todos os casos, inferior a $0,03 \mathrm{~m}$ e erro médio instantâneo observado foi de $0,06 \mathrm{~m}$.

Todavia, não foram encontradas soluções para o controle automático de direção e velocidade de veículos agrícolas que devem trafegar sincronizados. MAGALHÃES et al. (2008) descreveram um sistema de controle de posicionamento para auxiliar o sincronismo entre a colhedora de cana-de-açúcar e o veículo de transbordo, utilizando sensores de ultrassom para identificar a posição dos veículos e transmissores de rádio frequência para a comunicação entre operadores das máquinas que realizavam o ajuste da velocidade manualmente. O uso do sistema permitiu reduzir as perdas de matéria-prima em cerca de $60 \mathrm{~kg} \mathrm{ha}^{-1}$, comparado com o sistema desligado.

O presente trabalho teve por objetivo implementar e avaliar o desempenho de um sistema automático de controle de sincronismo de velocidade, desenvolvido em lógica fuzzy e analisar a viabilidade de sua utilização em operações de colheita que requerem o acompanhamento de veículos para recolhimento da biomassa junto à colhedora.

\section{MATERIAL E MÉTODOS}

Os testes com o sistema de controle de velocidade foram realizados no campo experimental da ESALQ, Escola Superior de Agricultura "Luiz de Queiroz”, Piracicaba, SP, durante os meses de junho e julho de 2010. Para o experimento, utilizou-se, como trator mestre, um Massey Ferguson modelo 291 equipado com motor Perkins modelo 1104C - 44T de 4 cilindros com aspiração natural e acionamento mecânico com $74 \mathrm{~kW}$ de potência e, como escravo, um trator Massey Ferguson modelo 5320 equipado com motor Perkins modelo 1006-6 de 6 cilindros com aspiração natural e acionamento mecânico com 88,2kW de potência, ambos trabalharam na faixa de rotação de 1800 a 2200rpm. O trator mestre foi instrumentado com computador de bordo, transmissor de radiofrequência com protocolo de comunicação ZigBee (XBee , MaxStream, Minnetonka), encoder (Hohner, série 58, Beamsville) instalado na roda com a finalidade de medir o deslocamento do trator e GPS (Garmin Corporation, série 18PC, Taiwan). No computador de bordo, foi 
instalado um programa mestre desenvolvido em Visual Basic utilizando o software Microsoft ${ }^{\circledR}$ Visual Studio ${ }^{\circledR}$ NET 2008 que, como entrada, recebe o valor da velocidade do GPS e os pulsos do encoder e, como saída, envia as informações de velocidade ao trator escravo. No trator escravo, foram instalados os mesmos componentes do trator mestre e também um motor de passo (TAK57H8604, Tekkno, Bauru) como atuador sobre o governador mecânico para controle da velocidade do trator.

Como sistema de controle, utilizou-se a equação fuzzy de velocidade modelada e simulada por BALDO (2011) como segue: $\mathrm{z}=\mathrm{a}+\mathrm{b}^{*} \mathrm{x}+\mathrm{c}^{*} \mathrm{y}+\mathrm{d}^{*} \mathrm{x}^{2}+$ $e^{*} y^{2}+f^{*} x^{*} y+g^{*} x^{3}+h^{*} y^{3} i^{*} x^{*} y^{2}+j^{*} x^{2 *} y$, em que: $z=$ Saída (atuação,\%); $\mathrm{x}=$ Entrada (diferença entre a velocidade do trator mestre e escravo); $\mathrm{y}=$ Entrada (erro da diferença de velocidade); $a$ até $\mathrm{j}=$ coeficientes dos termos dependentes da equação, determinados a partir do Toobox-Fuzzy do MatLAB (MatWorks, Versão 6.1).

Essa equação tem como entrada a diferença de velocidade entre o trator mestre e o trator escravo e a diferença do erro atual com o erro anterior. Como saída, o controlador fuzzy gera um sinal que representa a porcentagem de atuação na velocidade (Figura 1).

Para validar o sistema, primeiramente, realizaram-se testes somente com o trator escravo, utilizando, como sinal de entrada, valores préprogramados de velocidade. Nesse caso, aplicaramse funções degrau ascendente e descendente, uma com entrada de $\pm 0,14 \mathrm{~m} \mathrm{~s}^{-1}\left(0,5 \mathrm{~km} \mathrm{~h}^{-1}\right)$ e outra de $\pm 0,42 \mathrm{~m} \mathrm{~s}^{-1}\left(1,5 \mathrm{~km} \mathrm{~h}^{-1}\right)$, na faixa de $1,11 \mathrm{~m} \mathrm{~s}^{-1}\left(4,0 \mathrm{~km} \mathrm{~h}^{-1}\right)$ até $1,53 \mathrm{~m} \mathrm{~s}^{-1}\left(5,5 \mathrm{~km} \mathrm{~h}^{-1}\right)$. No segundo teste, utilizaram-se dois tratores, o mestre e o escravo, e a velocidade do mestre foi incrementada de $\pm 0,14 \mathrm{~m} \mathrm{~s}^{-1}$ ou de $\pm 0,42 \mathrm{~m} \mathrm{~s}^{-1}$ na faixa de operação de $1,11 \mathrm{~m} \mathrm{~s}^{-1}$ a $1,53 \mathrm{~m} \mathrm{~s}^{-1}$. Em ambos os casos, foram realizadas três repetições para cada teste.

A análise dos resultados consistiu na determinação do erro médio (EM), calculado pela soma dos módulos dos erros de previsão das velocidades e deslocamentos dos tratores, dividido pelo número de observações; o erro quadrático médio (EQM), que é a soma dos erros de previsão ao quadrado, dividido pelo número de observações; e o erro médio percentual (EMP), que é a diferença entre o valor medido e desejado no período, dividido pelo valor desejado e pelo número de observações.

\section{RESULTADO E DISCUSSÃO}

A figura 2 apresenta a simulação da variação de velocidade de entrada e a resposta do trator escravo para os testes realizados somente com um trator. Pelos gráficos, observa-se que o trator acompanhou a velocidade estabelecida, no entanto, é possível verificar flutuações sobre o valor da velocidade que ocorre principalmente em virtude do sistema de controle mecânico de injeção do motor diesel. A tabela 1 apresenta a estatística descritiva realizada com as diferenças entre a velocidade do trator escravo e a velocidade objetivo, para toda a faixa de teste. Verifica-se que, em todos os casos, o EQM não foi significativo e o maior EMP, em módulo, foi de 1,4\%. A máxima diferença de velocidade durante o percurso, em módulo, foi de $0,45 \mathrm{~m} \mathrm{~s}^{-1}$ nos testes realizados com a variação de velocidade de $1,53 \mathrm{~m} \mathrm{~s}^{-1}$ para $1,11 \mathrm{~m} \mathrm{~s}^{-1}$. Já nos teste com a função degrau de velocidade $0,14 \mathrm{~m} \mathrm{~s}^{-1}$, a máxima diferença de velocidade durante o percurso foi de $0,22 \mathrm{~m} \mathrm{~s}^{-1}$ na variação de $1,25 \mathrm{~m} \mathrm{~s}^{-1}$ para $1,39 \mathrm{~m} \mathrm{~s}^{-1}$.

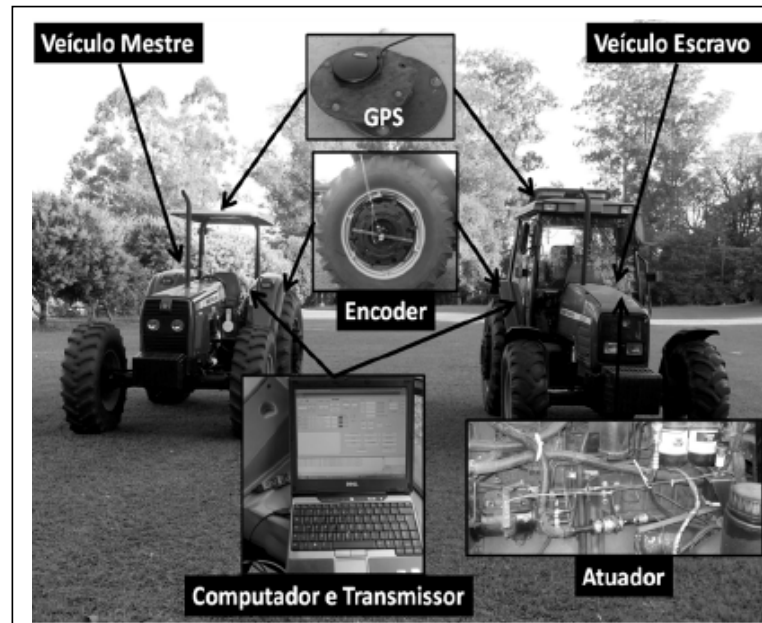

(a)

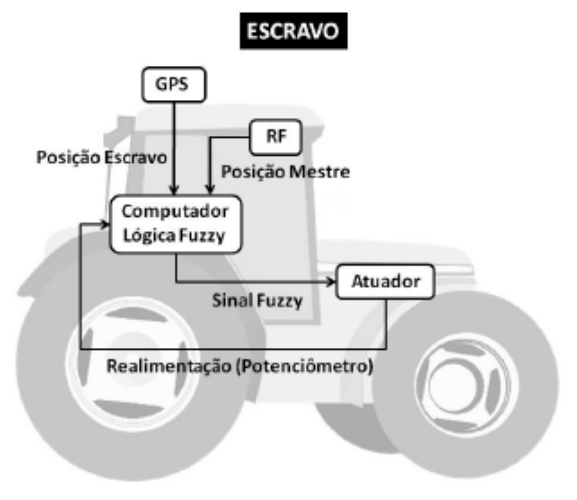

(b)

Figura 1 - a) Componentes de controle e atuação instalados nos tratores mestre e escravo; b) Sistema de controle de velocidade instalado no trator escravo. 


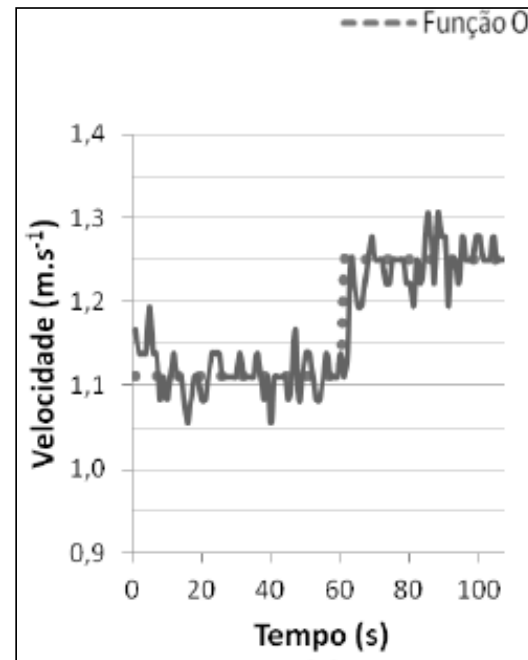

(a)

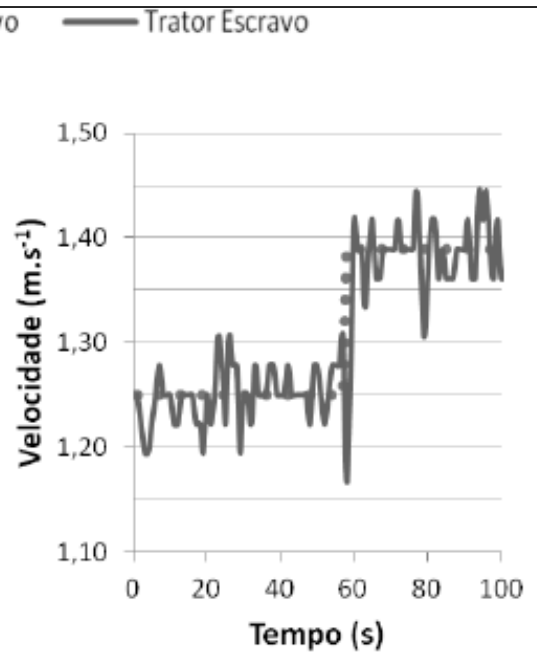

(b)

Figura 2 - Velocidade em função do tempo do trator escravo quando foi simulando a variação da velocidade do trator mestre; a) aumentando a velocidade do trator mestre de $1,1 \mathrm{~m} \mathrm{~s}^{-1}$ para $1,3 \mathrm{~m} \mathrm{~s}^{-1}$; b) aumentando a velocidade do trator mestre de $1,3 \mathrm{~m} \mathrm{~s}^{-1}$ para $1,4 \mathrm{~m} \mathrm{~s}^{-1}$.

A figura 3 apresenta a resposta do sistema com os dois tratores variando a velocidade do trator mestre de um valor de referência de $1,11 \mathrm{~m} \mathrm{~s}^{-1}$ para um valor de referência de $1,25 \mathrm{~m} \mathrm{~s}^{-1}$. Pode-se observar nessa figura que tanto a velocidade do trator mestre quanto a do escravo variam no tempo e, para poder analisar de forma mais clara os resultados, extraíram-se as médias dos valores das velocidades em regime antes e depois da função degrau, analisando assim os períodos de regime do sistema.

O gráfico de velocidade média do trator mestre e escravo é apresentado na figura 4. Observase que o trator escravo acompanhou o mestre na variação da velocidade, porém verifica-se que as velocidades médias se diferem da mesma forma apresentada no primeiro teste. A tabela 2 apresenta o total do deslocamento dos tratores, bem como o erro de deslocamento causado pela diferença entre o deslocamento total do trator mestre e do escravo, medido pelos encoders. Pela tabela, observa-se que o EMP é aleatório até mesmo dentro de cada teste, variando, por exemplo, de $-0,3 \%$ a $1,9 \%$ quando o acréscimo da velocidade do trator mestre foi de 1,25 para 1,39 $\mathrm{m} \mathrm{s}^{-1}$. O maior EMP de deslocamento em todos os testes foi de 2,8\%. Esses resultados indicam que o sistema de controle de velocidade desenvolvido precisa ainda ser aprimorado para atender às necessidades reais de campo, onde o erro máximo admitido seria na faixa de $0,5 \%$. Os erros gerados são decorrentes principalmente do sistema de acelerador do trator, que é acionado por governadores mecânicos. Esses erros poderiam ser corrigidos com o auxílio de sensores de

Tabela 1 - Análise estatística das diferenças entre a função objetivo de velocidade e a obtida pelo trator escravo no decorrer do percurso.

\begin{tabular}{|c|c|c|c|c|c|c|c|c|}
\hline & 1,11 & 1,25 & 1,39 & 1,53 & 1,39 & 1,25 & 1,11 & 1,53 \\
\hline & 1,25 & 1,39 & 1,53 & 1,39 & 1,25 & 1,11 & 1,53 & 1,11 \\
\hline Erro médio (EM) $\left(\mathrm{m} \mathrm{s}^{-1}\right)$ & NS & NS & NS & $-0,01$ & NS & NS & NS & $-0,01$ \\
\hline Erro padrão (m s $\left.{ }^{-1}\right)$ & NS & NS & NS & NS & NS & NS & NS & 0,01 \\
\hline Desvio padrão $\left(\mathrm{m} \mathrm{s}^{-1}\right)$ & 0,03 & 0,03 & 0,03 & 0,03 & 0,03 & 0,03 & 0,05 & 0,07 \\
\hline $\operatorname{EQM}\left(\mathrm{m} \mathrm{s}^{-1}\right)$ & NS & NS & NS & NS & NS & NS & NS & NS \\
\hline EMP (\%) & 0,02 & 0,22 & 0,1 & $-0,45$ & $-0,4$ & $-0,36$ & 0,58 & $-1,37$ \\
\hline Mínimo $\left(\mathrm{m} \mathrm{s}^{-1}\right)$ & 0,08 & $-0,1$ & $-0,1$ & $-0,14$ & $-0,2$ & $-0,17$ & $-0,1$ & $-0,44$ \\
\hline Máximo $\left(\mathrm{m} \mathrm{s}^{-1}\right)$ & 0,14 & 0,22 & 0,11 & 0,06 & 0,08 & 0,08 & 0,41 & 0,06 \\
\hline
\end{tabular}

EQM = erro quadrático médio EMP = erro médio percentual NS = número não significativo.

Ciência Rural, v.42, n.2, fev, 2012. 


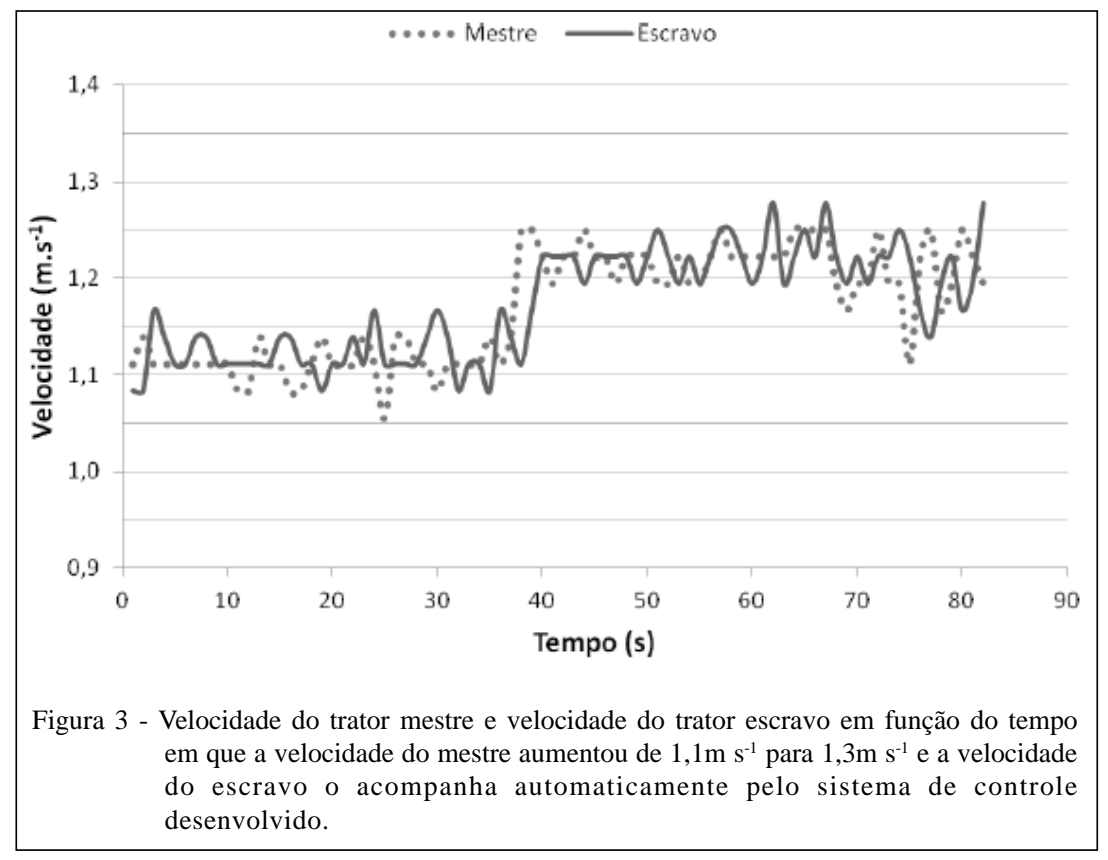

presença instalados na lateral do transbordo de forma a indicar o desalinhamento das máquinas e retroalimentar o sistema de controle, ou utilizando-se tratores equipados com motores eletrônicos.

\section{CONCLUSÃO}

Os testes de campo comprovaram que o sistema de controle utilizando lógica fuzzy como controlador PID pode ser utilizado para controlar a velocidade de deslocamento de dois tratores que trabalham sincronizados durante operações agrícolas. O sistema atuou dentro dos limites estabelecidos para velocidades convencionais de colheita entre $1,0 \mathrm{~m} \mathrm{~s}^{-1} \mathrm{e}$ $1,4 \mathrm{~m} \mathrm{~s}^{-1}$, mantendo a diferença entre as velocidades dos tratores menor que $0,01 \mathrm{~m} \mathrm{~s}^{-1}$ com EMP de velocidade menor que $1,4 \%$. A análise de deslocamento apresentou um EMP variando de 0,2\% a 2,8\%,

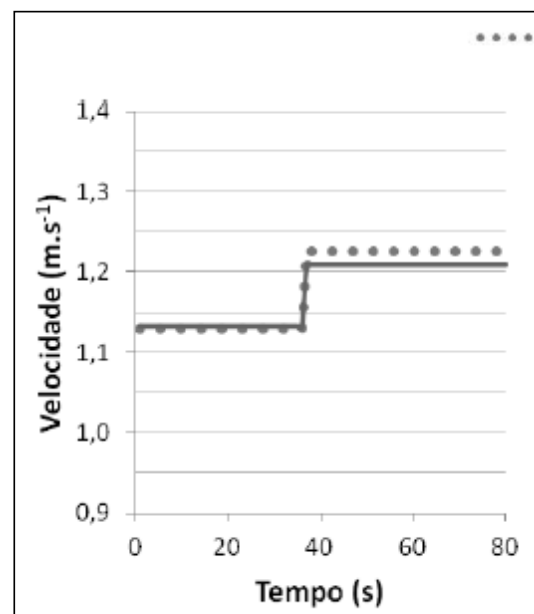

(a)

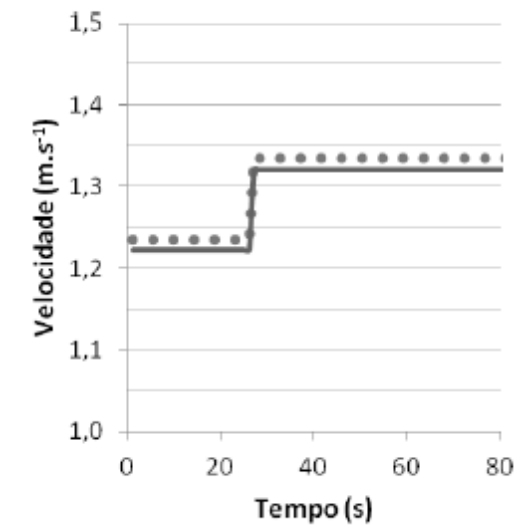

(b)

Figura 4 - Velocidade média do trator mestre e do trator escravo, variando a velocidade do trator mestre; a) aumentando a velocidade do trator mestre de $1,1 \mathrm{~m} \mathrm{~s}^{-1}$ para $1,3 \mathrm{~m} \mathrm{~s}^{-1}$; b) aumentando a velocidade do trator mestre de $1,3 \mathrm{~m} \mathrm{~s}^{-1}$ para $1,4 \mathrm{~m} \mathrm{~s}^{-1}$.

Ciência Rural, v.42, n.2, fev, 2012. 
Tabela 2 - Erro de deslocamento entre o trator mestre e escravo no decorrer do percurso para os testes realizados com os dois tratores.

\begin{tabular}{|c|c|c|c|c|}
\hline Velocidade $\left(\mathrm{m} \mathrm{s}^{-1}\right)$ & & TESTE 1 & TESTE 2 & TESTE 3 \\
\hline \multirow{4}{*}{$1,11-1,25$} & Total deslocamento (m) & 94,8 & 115,6 & 117,7 \\
\hline & Erro médio (m) & NS & NS & 0,02 \\
\hline & EQM (m) & NS & NS & NS \\
\hline & EMP (\%) & $-0,22$ & $-0,16$ & 1,50 \\
\hline \multirow{4}{*}{$1,25-1,39$} & Total deslocamento (m) & 107,6 & 128,0 & 107,6 \\
\hline & Erro médio (m) & NS & 0,02 & 0,03 \\
\hline & EQM (m) & NS & NS & NS \\
\hline & EMP (\%) & $-0,35$ & 1,67 & 1,94 \\
\hline \multirow{4}{*}{$1,39-1,53$} & Total deslocamento (m) & 107,6 & 128,0 & 132,1 \\
\hline & Erro médio (m) & 0,01 & 0,02 & 0,01 \\
\hline & EQM (m) & NS & NS & NS \\
\hline & EMP (\%) & 0,80 & 1,70 & 0,71 \\
\hline \multirow{4}{*}{$1,53-1,39$} & Total deslocamento (m) & 112,9 & 118,6 & 110,1 \\
\hline & Erro médio (m) & $-0,01$ & 0,01 & 0,02 \\
\hline & EQM (m) & NS & NS & NS \\
\hline & EMP (\%) & $-0,81$ & 0,74 & 1,54 \\
\hline \multirow{4}{*}{$1,39-1,25$} & Total deslocamento (m) & 114,3 & 114,5 & 113,2 \\
\hline & Erro médio (m) & $-0,01$ & NS & 0,01 \\
\hline & EQM (m) & 0,01 & NS & NS \\
\hline & EMP (\%) & $-1,09$ & $-0,35$ & 0,32 \\
\hline \multirow{4}{*}{$1,25-1,11$} & Total deslocamento (m) & 116,6 & 117,8 & 114,0 \\
\hline & Erro médio (m) & NS & $-0,01$ & $-0,01$ \\
\hline & EQM (m) & NS & NS & NS \\
\hline & EMP (\%) & $-0,20$ & $-0,82$ & 0,86 \\
\hline \multirow{4}{*}{$1,11-1,53$} & Total deslocamento (m) & 112,4 & 112,9 & 120,6 \\
\hline & Erro médio (m) & 0,01 & 0,01 & $-0,02$ \\
\hline & EQM (m) & 0,01 & 0,01 & 0,01 \\
\hline & EMP (\%) & 0,26 & 0,42 & $-1,81$ \\
\hline \multirow{4}{*}{$1,53-1,11$} & Total deslocamento (m) & 115,5 & 122,7 & 116,2 \\
\hline & Erro médio (m) & 0,01 & $-0,02$ & $-0,06$ \\
\hline & EQM (m) & 0,01 & 0,01 & 0,08 \\
\hline & EMP (\%) & 0,40 & $-2,02$ & $-2,85$ \\
\hline
\end{tabular}

EQM = erro quadrático médio EMP = erro médio percentual NS = número não significativo.

mostrando que, para uma aplicação real de campo, seria necessário ainda um aprimoramento do sistema, implementando sensores de retroalimentação que corrigiriam os erros propagados de deslocamento.

\section{AGRADECIMENTOS E APRESENTAÇÃO}

Os autores agradecem ao Financiamento de Estudos e Projetos (FINEP) e à Fundação de Amparo à Pesquisa do
Estado de São Paulo (FAPESP), pelo financiamento do projeto de pesquisa, e ao Conselho de Desenvolvimento Científico e Tecnológico (CNPq), pela concessão da bolsa de doutoramento ao primeiro autor, à Escola Superior de Agricultura "Luiz de Queirós” (Esalq), Universidade Federal de São Paulo (USP), pela cessão dos tratores e área experimental, e à Faculdade de Engenharia Agrícola (FEAGRI), Universidade Estadual de Campinas (UNICAMP), pela disponibilização do laboratório e pelas facilidades proporcionadas à realização deste trabalho.

Este trabalho faz parte da tese de doutorado defendida pelos autores na FEAGRI/UNICAMP.

Ciência Rural, v.42, n.2, fev, 2012. 


\section{REFERÊNCIAS}

BALDO, R.F.G. Controle da velocidade e da direção entre dois veículos agrícolas. 2011. 100f. Tese (Doutorado em Máquinas Agrícolas) - Curso de pós-graduação em Engenharia Agrícola, Faculdade de Engenharia Agrícola, UNICAMP, Campinas, SP. Disponível em: <http://cutter.unicamp.br/ document/?code=000784212>. Acesso em: 28 out. 2011.

BERGLUND, S.; BUICK, R. Guidance and automated steering drive resurgence in precision farming. In: EUROPEAN CONFERENCE ON PRECISION AGRICULTURE, 5., 2005, Uppsala. Proceedings... Uppsala: John Stafford, 2005. p.39-45.

FOSTER, C.A. et al. Automatic velocity control of a self-propelled windrower. Computers and Electronics in Agriculture, v.47, p.41-58, 2005. Disponível em: <http://linkinghub.elsevier.com/ retrieve/pii/S0168169904001206>. Acesso em: 03 fev. 2011. doi: 10.1016/j.compag.2004.10.001

HAGRAS, H. et al. Outdoor mobile robots learning and adaptation. IEEE Robotics \& Automation Magazine, v.8, n.3, p.53-69, 2001. Disponível em: <http://csa.com/partners/ viewrecord.php?requester=gs\&collection=TRD\&recid=A0211464AH>. Acesso em: 03 fev. 2011.

KISE, M. et al. A stereovision-based crop row detection method for tractor-automated guidance. Biosystems Engineering, v.90, n.4, p.357-367, 2005. Disponível em: <http:// w w w.sciencedirect.com/science/article/pi i / S1537511004002260>. Acesso em: 28 out. 2011. doi:10.1016/ j.biosystemseng.2004.12.008.

KODAGODA, K.R. et al. Fuzzy speed and steering control of an AGV. IEEE Transactions on Control Systems Technology, v.10, n.1, p.112-120, 2002. Disponível em: <http:// ieeexplore.ieee.org/xpl/freeabs_all.jsp?arnumber=974344>. Acesso em: 28 out. 2011. doi: 10.1109/87.974344.

MAGALHÃES, P.S.G. et al. Sistema de sincronismo entre a colhedora de cana-de-açúcar e o veículo de transbordo. Engenharia Agrícola, v.28, n.2, p.274-282, 2008. Disponível em: <http://www.scielo.br/ scielo.php?script=sci_arttext\&pid=S0100-69162008000200008>. Acesso em: 28 out. 2011.doi: 10.1590/S0100-69162008000200008.

ROVIRA-MÁS, F. Sensor architecture and task classification for agricultural vehicles and environments. Sensors, v.10, n.12, p.11226-11247. Disponível em: <http://www.mdpi.com/ 1424-8220/10/12/11226>. Acesso em: 28 out. 2011. doi: $10.3390 / \mathrm{s} 101211226$.

SUBRAMANIAN, V. et al. Development of machine vision and laser radar based autonomous vehicle guidance systems for citrus grove navigation. Computers and Electronics in Agriculture, v.53, n.2, p.130-143, 2006. Disponível em: <http:// www.sciencedirect.com/science/article/pii/S016816990600069X>. Acesso em: 28 out. 2011. doi: 10.1016/j.compag.2006.06.001. 